\title{
Distale Humerusfrakturen beim Kind
}

Stefan Nuber, Johannes Plath, Stefan Förch, Edgar Mayr

\section{Epidemiologie}

Circa 10\% der Frakturen im Kindes- und Jugendalter betreffen den distalen Humerus. In ca. 70\% der Fälle handelt es sich hierbei um extraartikuläre suprakondyläre Oberarmfrakturen. Die charakteristische Altersgruppe für diese Fraktur liegt am Übergang zum Grundschulalter (Altersgipfel 6. Lebensjahr). In etwa $12 \%$ treten isolierte Abrissfrakturen des Epicondylus ulnaris und hiervon in 75\% der Fälle in Kombination mit einer Ellenbogenluxation auf. Bei einem Altersgipfel von 11 Jahren sind vor allem ältere Kinder und Adoleszenten betroffen. In 17\% der Fälle liegt eine Condylus-radialis-Fraktur vor, die im Wesentlichen im Kindergartenalter auftritt (Altersgipfel 4. Lebensjahr). Condylus-ulnaris- und transkondyläre Y-Frakturen sind sehr selten, treten vor allem bei Adoleszenten kurz vor Wachstumsabschluss auf und machen zusammen weniger als $1 \%$ der Frakturen am distalen Humerus aus $[6,7,10]$.

\section{Anatomie/Wachstumsprognose/ Systematik}

Der distale Humerus und der proximale Unterarm unterliegen im Wachstum einer zeitlich typischen, jedoch trotzdem unregelmäßigen Entwicklung der vorhandenen 6 Knochenkerne. Dies betrifft auch den funktionellen Verschluss der distalen Humerusfuge (s. - Abb. 1). Dieser Umstand kann bei der Röntgendiagnostik zu Schwierigkeiten bei der Diagnosestellung führen. Es kommt daher immer wieder zu fälschlicherweise angenommenen Frakturen oder sogar aseptischen Knochennekrosen (z. B. beim Trochlea-humeri-Knochenkern).

Beim therapeutischen Vorgehen und der Prognose muss prinzipiell zwischen den rein metaphysären Frakturen (suprakondyläre Humerusfraktur und Abrissfraktur des Epicondylus ulnaris) sowie den gelenkbeteiligenden Epiphysenfrakturen (Frakturen des Condylus radialis oder Condylus ulnaris und transkondyläre Y-Frakturen) unterschieden werden [1,2]. Dislozierte Gelenkfrakturen bedürfen einer anatomischen Gelenkrekonstruktion, um Präarthrosen zu vermeiden. Dagegen können bei metaphysären Frakturen Dislokationen innerhalb bestimmter Toleranzgrenzen und Altersgruppen akzeptiert werden. Grundsätzlich ist anzumerken, dass das Wachstums- potenzial der distalen Humerusepiphyse lediglich mit einem Anteil von 20\% am Gesamtwachstum des Humerus beteiligt ist. Deshalb ist das Korrekturpotenzial insgesamt gering [5].

\section{Suprakondyläre Humerusfraktur}

\section{Verletzungsmechanismus/ klinisches Bild/Diagnostik}

Die suprakondylären Humerusfrakturen entstehen meist durch Sturz auf den ausgestreckten Arm (Extensionsfraktur). Sehr selten sind Flexionsfrakturen durch einen Sturz direkt auf das Ellenbogengelenk (s. - Abb. 2) [10].

Das klinische Bild hängt vom Ausmaß der Dislokation ab. Zur Anamnese gehört ein verletzungsadäquates Trauma. Das Ellenbogengelenk ist geschwollen, bei deutlicher Dislokation ist die Fehlstellung des Gelenks nicht zu übersehen. Geachtet werden muss auf die Durchblutung (Pulsqualität im Seitenvergleich) und ein mögliches peripheres sensomotorisches Defizit. In der Akutphase bereitet es in der Praxis häufig Schwierigkeiten, bei jungen Kindern einen adäquaten neurologischen Befund zu erheben, was ggf. entsprechend dokumentiert werden sollte.

Die Akutdiagnostik beinhaltet eine Röntgenuntersuchung in 2 Ebenen, wobei in der Realität aufgrund der in aller Regel anliegenden Oberarmschiene und der Schmerzsymptomatik selten eine echte a.-p. Aufnahme in Ellenbogenstreckung sowie eine exakte seitliche Projektion in $90^{\circ}$ Beugestellung erreicht werden. Dies kann zu Schwierigkeiten bei der Interpretation der Röntgenbilder führen.

Vor allem bei undislozierten Frakturen muss man auf indirekte Frakturzeichen wie das sog. Fat-Pad-Zeichen (ventral und/oder dorsal des distalen Humerus) als Ausdruck eines intraartikulären Ergusses (s. D Abb. 3) geachtet werden. Eine Sonografie, eine CT oder eine MRT werden zur Diagnosefindung in aller Regel nicht benötigt.

\section{Klassifikation der suprakondylären Humerusfraktur}

Am häufigsten in der Praxis angewendet und bewährt hat sich die therapiebezogene Klassifikation nach Laer und Mitarbeitern (s. A Abb.4) [7, 10]. Typ I beschreibt die nicht dislozierte Fraktur. Beim Typ II besteht eine Disloka- 


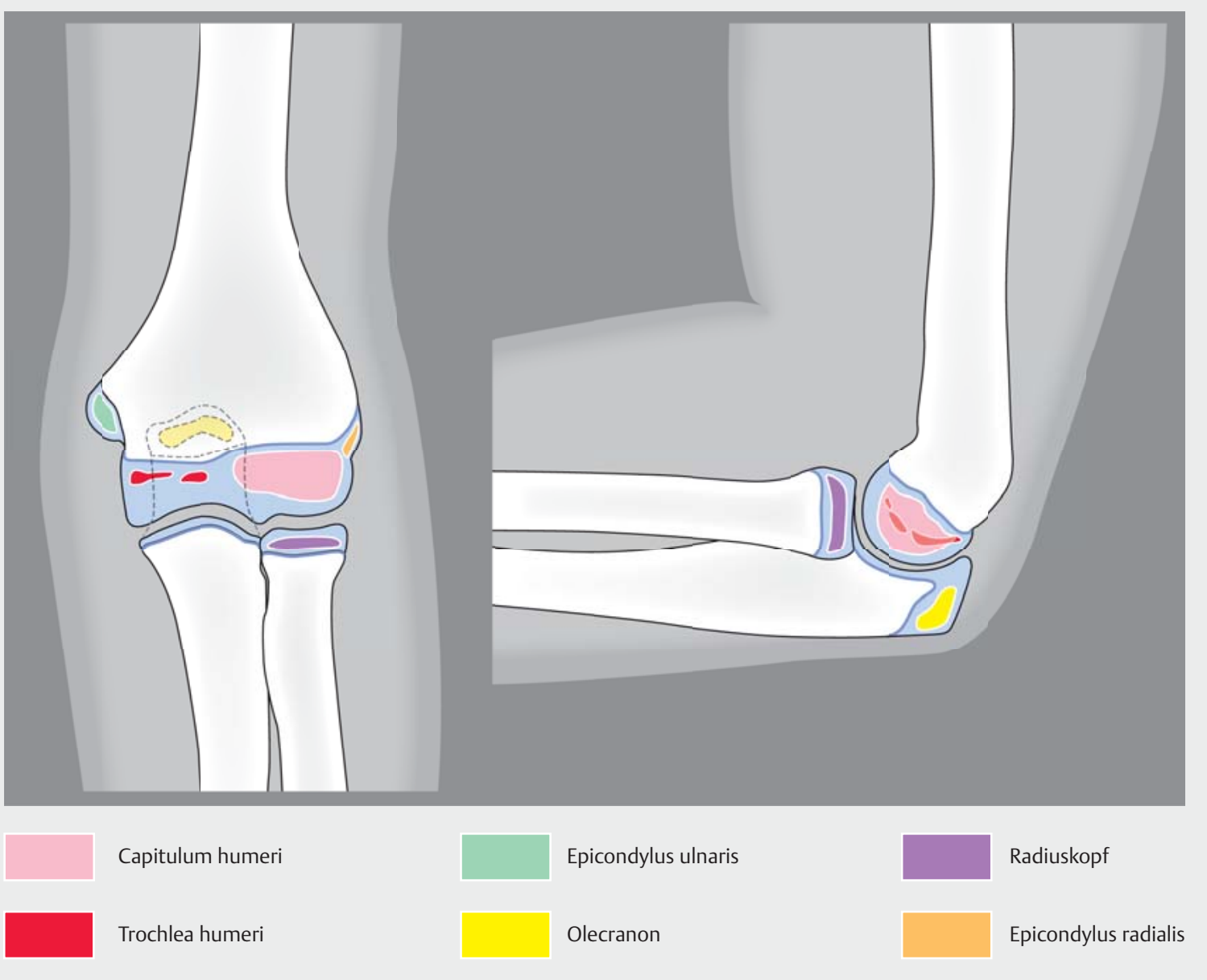

- Abb. 1 Knochenkerne am kindlichen Ellenbogen.
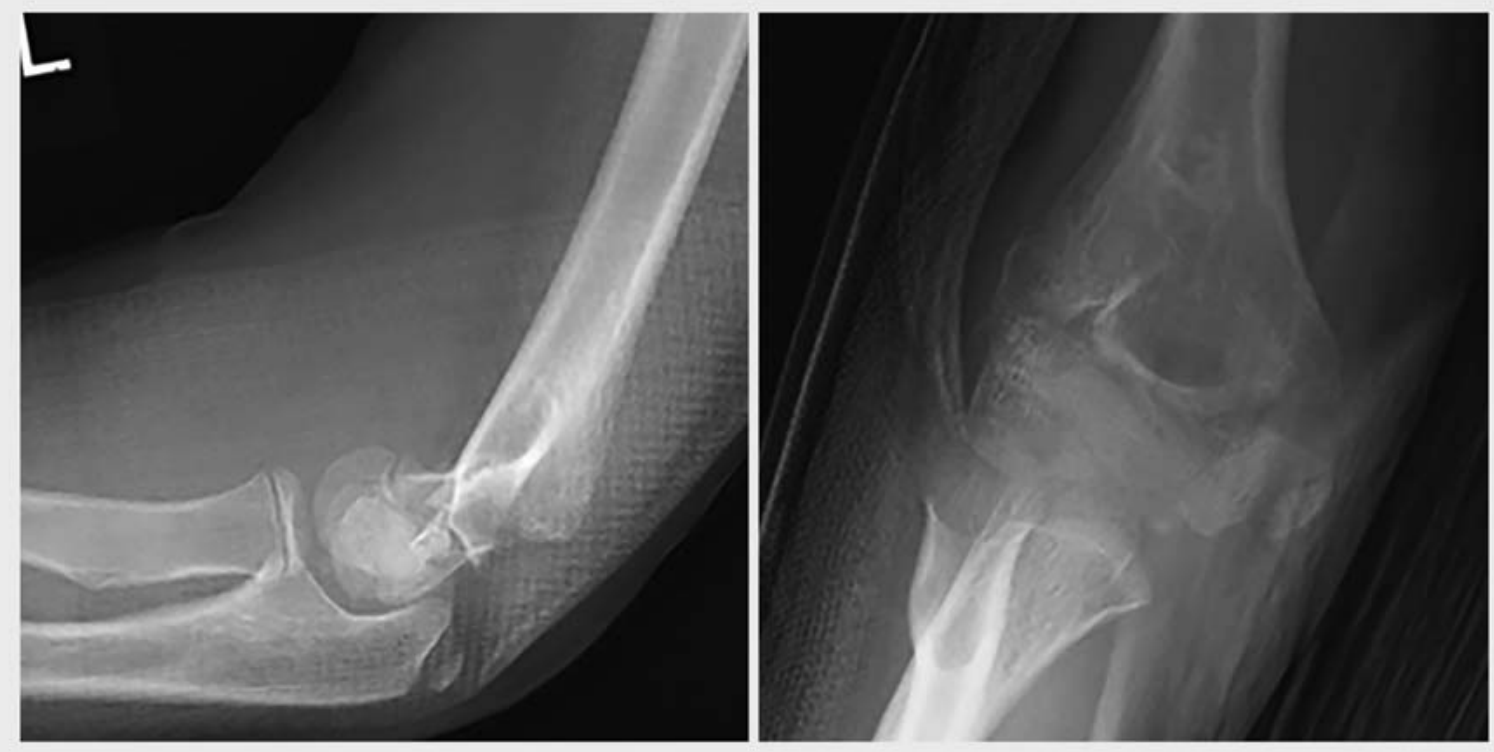

- Abb. 2 Kindliche suprakondyläre Flexionsfraktur in 2 Ebenen. 


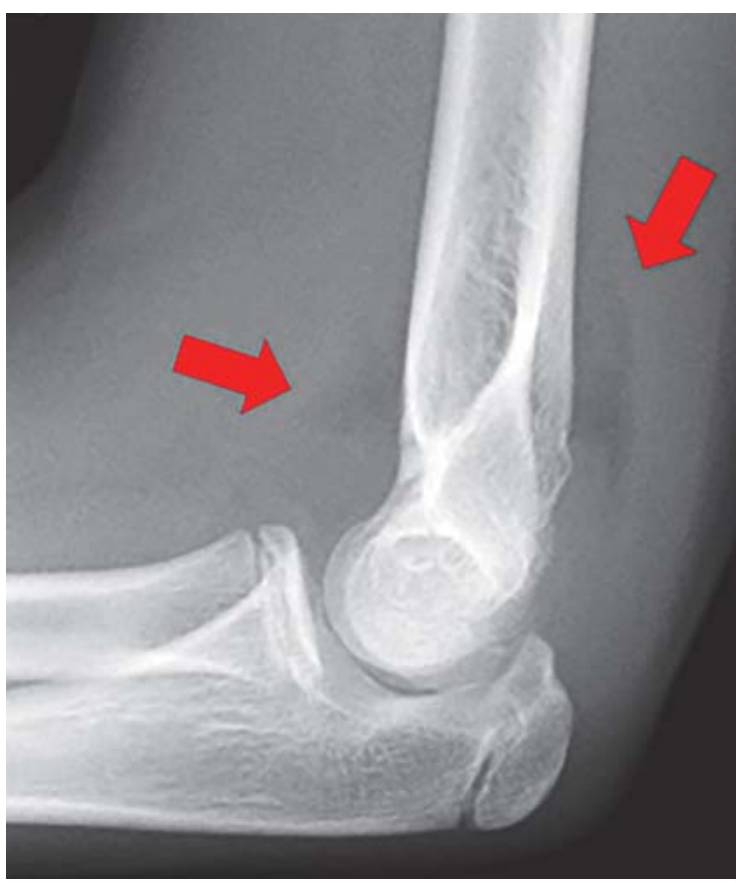

- Abb. 3 Positives ventrales und dorsales Fat-Pad-Zeichen (Pfeile) bei undislozierter suprakondylärer Oberarmfraktur.

tion in der sagittalen Ebene. In 98\% der Fälle handelt es sich um eine Antekurvation (Extensionsfraktur), nur sehr selten besteht eine Rekurvation (Flexionsfraktur). Bei den Typ-II-Extensionsfrakturen wird unterschieden zwischen den stabilen (höchstens 20-30 Antekurvation) und den drohend instabilen (Antekurvation $>30^{\circ}$, ggf. mit Seitverschiebung) Verletzungen. Typ III beinhaltet Frakturen mit Dislokation in 2 Ebenen mit Rotationsfehler. Beim Typ IV besteht die Dislokation in 3 Ebenen (komplett disloziert Fraktur ohne knöchernen Kontakt).

\section{Therapie/Komplikationen/Nachbehandlung}

Ziel der Behandlung ist die anatomische Stellung/Reposition der Fraktur und der Erhalt bzw. die Etablierung einer stabilen Fraktursituation [1]. Typ-I-Verletzungen gelten grundsätzlich als stabil und können immer konservativ behandelt werden. Es erfolgt neben einer adäquaten Schmerztherapie die Ruhigstellung in einer Oberarmschiene oder einer Blount-Schlinge für 2-3 Wochen. Stabile Typ-II-Frakturen können ebenfalls konservativ behandelt werden. Bei sehr jungen Kindern (max. bis zum 5.6 . Lebensjahr) kann eine Spontankorrektur in der seitlichen Ebene (Antekurvation) von maximal $20^{\circ}$ in das Behandlungsregime miteingerechnet werden. Die übrigen Typ-II-Verletzungen bedürfen einer Redressierung. Entweder wird die Reposition (mittels Hyperflexion) der Antekurvationsfehlstellung in Kurznarkose mit anschließender Ruhigstellung im Spitzwinkelgips/-Dynacast durchgeführt oder es erfolgt durch einen anfangs täglich nachgezogenen Cuff-and-Collar-Verband über mehrere Tage

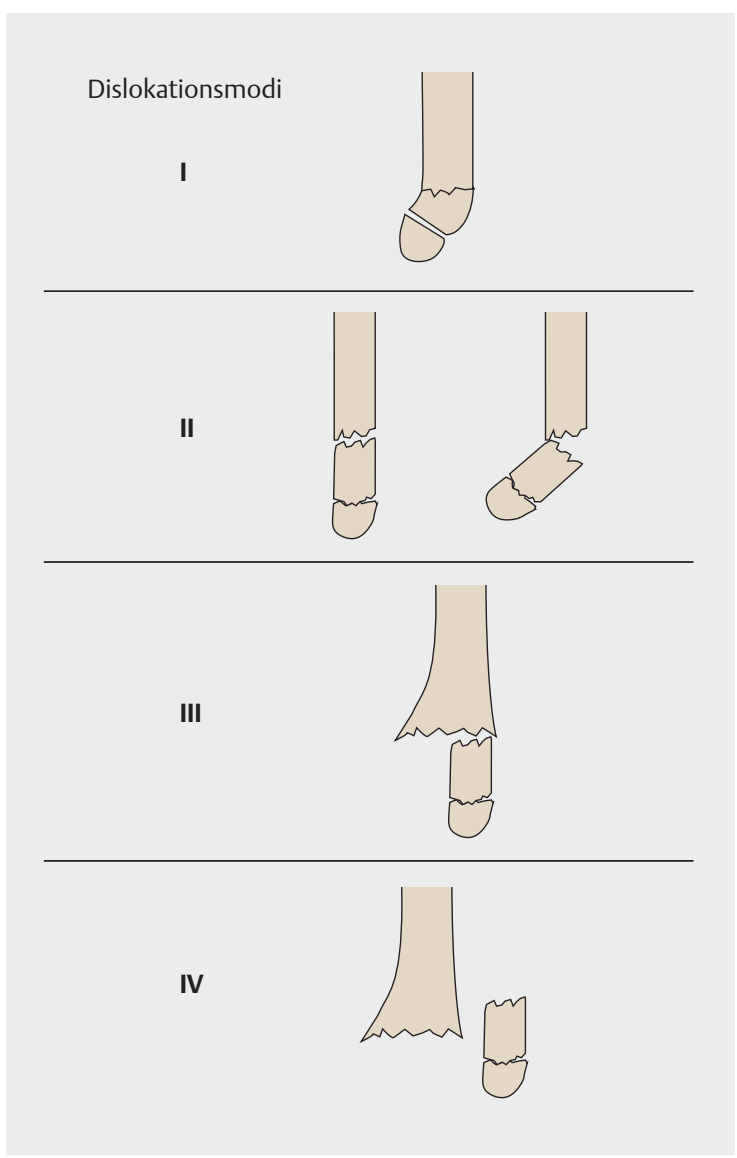

- Abb. 4 Klassifikation der suprakondylären Oberarmfrakturen nach Lutz v. Laer et al. 2002. Quelle: von Laer L, Kraus R, Linhart W. Verletzungsformen und Klassifikationen. In: von Laer L, Kraus R, Linhart W, Hrsg. Frakturen und Luxationen im Wachstumsalter. 6. Aufl. Stuttgart: Thieme; 2012: 143.

die Reposition der Fraktur durch die zunehmende Spitzwinkelstellung mit mindestens $120^{\circ}$ (s. Abb.5a-e). Bei Typ-II-Frakturen sollte eine Stellungskontrolle zum Ausschluss sekundärer Dislokationen (Rotationsfehler) nach ca. 5 Tagen erfolgen. Relevant dislozierte Typ-IIFrakturen sowie die Typ-III- und -IV-Frakturen werden operativ behandelt. Zum einen liegen hier eine relevante Instabilität und zum anderen entsprechende Achs- und Rotationsfehlstellungen vor, die ohne entsprechende Reposition und Retention zu wesentlichen Wachstumsstörungen mit schicksalhafter Ausbildung vor allem eines Cubitus varus führen können [7,9]. Grund hierfür ist die Abhängigkeit der Kontaktfläche der Fraktur von der Lage der Drehachse und dem Ausmaß einer Fehlrotation. Bei zentraler Drehachse nimmt mit zunehmender Rotation die Kontaktfläche der Fragmente gegeneinander linear bis $0 \%$ bei $70^{\circ}$ Fehlrotation ab. So beträgt die Auflagefläche schon bei einer Fehlrotation von $20^{\circ}$ weniger als $50 \%$. Unterschreitet die Kontaktfläche ein kritisches Minimum, kommt es aufgrund fehlender Abstützung zu einem Kol- 

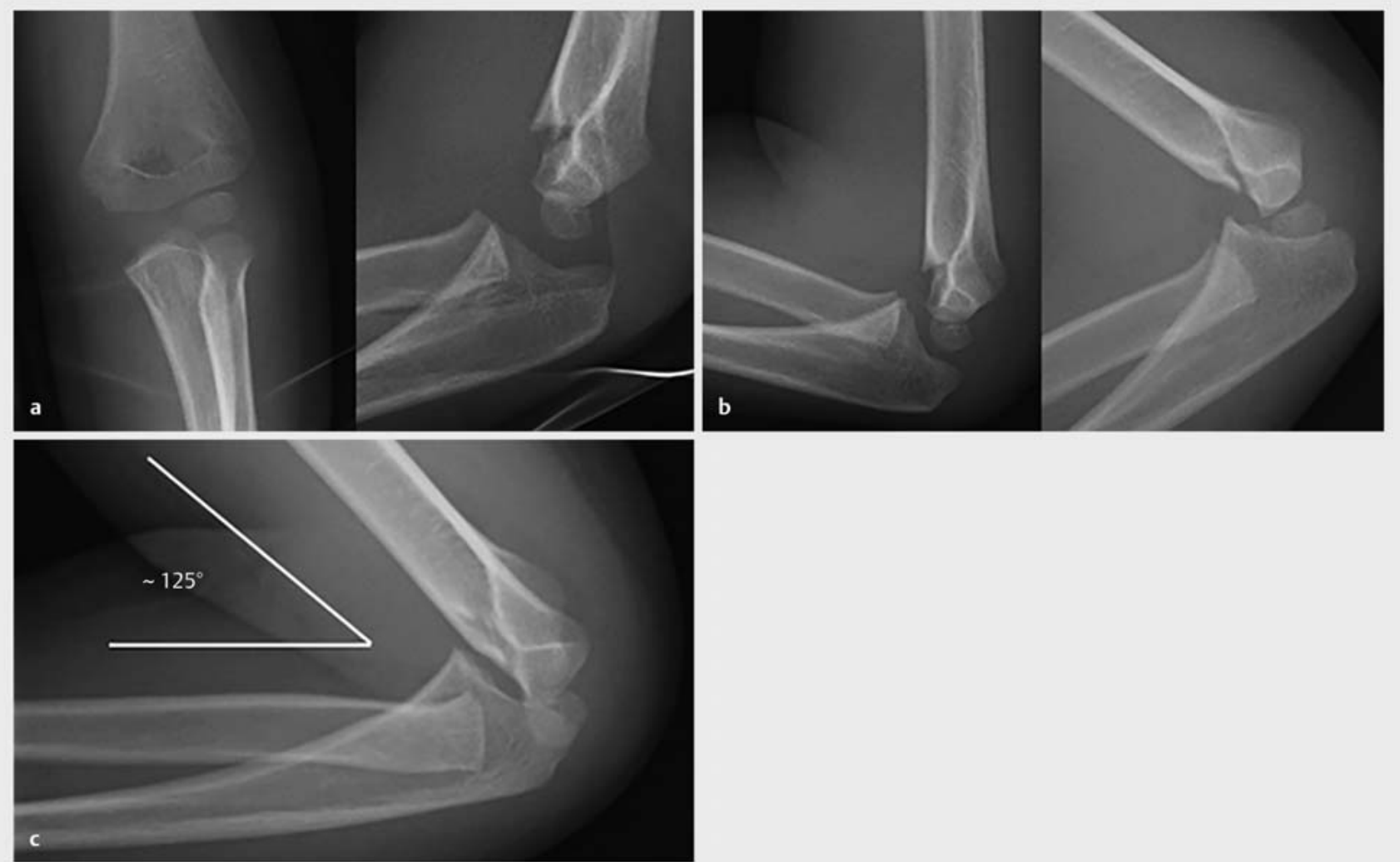

b

- Abb. 5 Redressierung einer suprakondylären Oberarmfraktur Typ II nach Lutz v. Laer über Cuff and Collar: a und b Unfallbilder, c nach 3 Tagen, d nach 6 Tagen, e nach 9 Tagen.

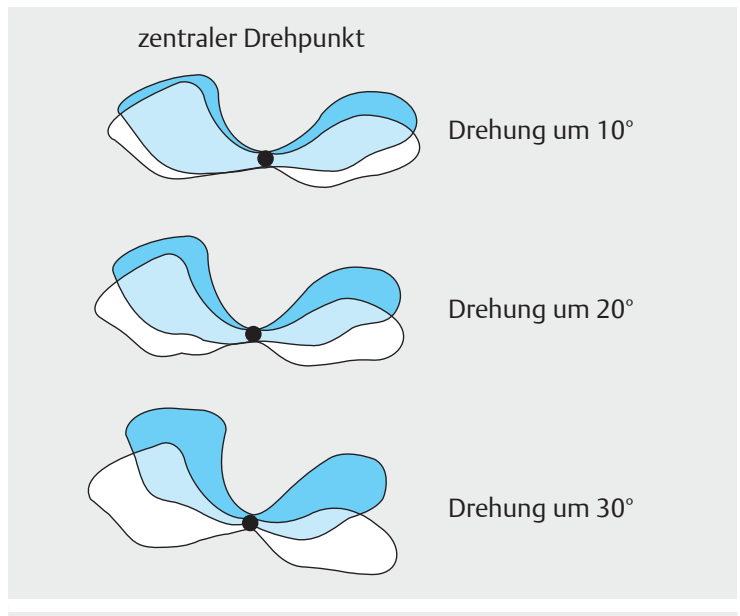

- Abb. 6 Abhängigkeit der Kontaktfläche (dunkel schraffiert) der Fraktur bei zentraler Drehachse und dem Ausmaß einer Fehlrotation (oben bei $10^{\circ}$, mittig bei $20^{\circ}$, unten bei $30^{\circ}$ ). Quelle: von Laer L, Kraus R, Linhart W. Probleme und Komplikationen. In: von Laer L, Kraus R, Linhart W, Hrsg. Frakturen und Luxationen im Wachstumsalter. 6. Aufl. Stuttgart: Thieme; 2012: 145-149. laps des ulnaren Pfeilers nach proximal und somit zu einer Varisierung der Ellenbogenachse (s. - Abb. 6) [7].

\section{Merke}

Relevant dislozierte suprakondyläre Typ-II- sowie die Typ-III- und -IV-Frakturen werden operativ behandelt!

In der Praxis hat sich die gekreuzte K-Draht-Spickung etabliert (s. - Abb. 7). In Intubationsnarkose und Rückenlage mit Auslagerung des betroffenen Armes auf ein Armbänkchen wird zunächst die Fraktur in aller Regel mittels Traktion, Flexion und Pronation geschlossen reponiert und mittels Bildwandler im seitlichen Strahlengang kontrolliert. Nach dem sterilen Abwaschen und Abdecken erfolgt unter Halten der Reposition zunächst die Platzierung des radialen K-Drahtes, der perkutan über eine Stichinzision vom immer zu tastenden Epicondylus radialis aus unter seitlicher Bildwandlerkontrolle bikortikal vorgebohrt wird. Beim rein perkutanen Vorgehen auf der ulnaren Seite unter Hyperflexion im Ellenbogengelenk kommt es in bis zu 15\% der Fälle zu einer Verletzung des N. ulnaris [3]. Deshalb machen wir für den ulnaren KDraht einen ca. $2 \mathrm{~cm}$ langen Zugang über dem Epicondylus ulnaris. Der N. ulnaris wird, soweit notwendig, lokali- 

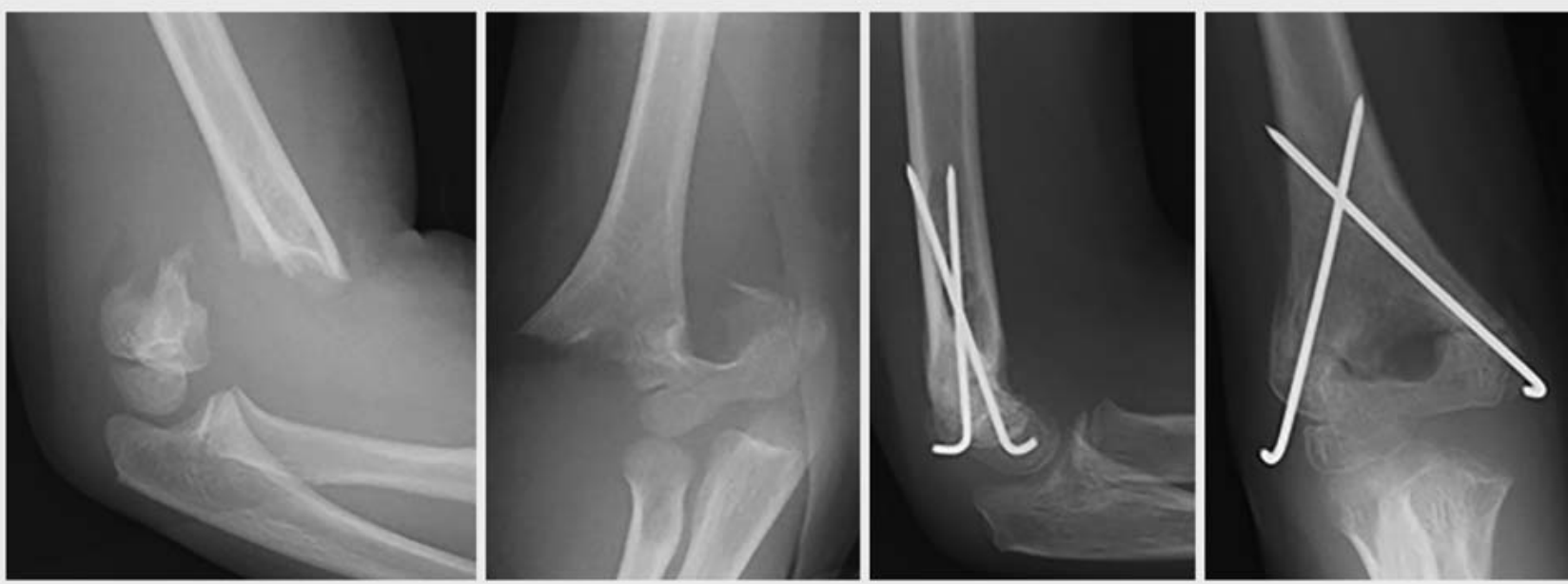

- Abb. 7 Suprakondyläre Oberarmfraktur Typ IV nach Lutz v. Laer: präoperative und postoperative Röntgenbilder in 2 Ebenen nach geschlossener Reposition und gekreuzter K-Draht-Spickung.

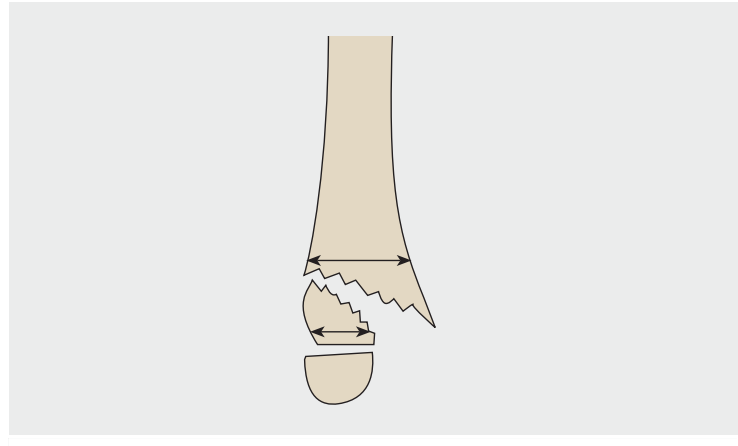

- Abb. 8 Schematische Darstellung eines Rotationssporns mit Kalibersprung bei einer suprakondylären Oberarmfraktur.Quelle: von Laer L, Kraus R, Linhart W. Probleme und Komplikationen. In: von Laer L, Kraus R, Linhart W, Hrsg. Frakturen und Luxationen im Wachstumsalter. 6. Aufl. Stuttgart: Thieme; 2012: 145-149.

siert und mit dem Finger oder der Pinzette unter Nachlassen der Flexion im Ellenbogen nach dorsal weggehalten. Nun setzen wird den ulnaren K-Draht auf dem Epicondylus ulnaris mit Gewebeschutz an, führen wieder die entsprechende Reposition unter Flexion durch und Bohren wiederum bikortikal vor. Es ist darauf zu achten, dass sich die beiden K-Drähte proximal der Fraktur kreuzen. Damit wird eine sekundäre Verdrehung der Fraktur verhindert. Wir kürzen und biegen die distalen Drahtenden um, die dann subkutan versenkt werden. In der Literatur wird weiterhin auch das perkutane Belassen der Drahtenden als mögliche Alternative ohne wesentliche Gefahr der Pin-Track-Infektion angegeben [8]. Sollte nach dem 2. geschlossenen Repositionsversuch keine anatomische
Stellung erzielt sein, wird die Umlagerung in Bauchlage mit anschließender offener Reposition empfohlen. Intrawie auch postoperativ sollte die seitliche Ebene im radioulnaren Strahlengang durchleuchtet bzw. geröntgt werden. Hiermit entgeht man der Gefahr, dass milde Rotationsfehler $\left(<20^{\circ}\right)$ durch Überlagerung vom Condylus radialis verdeckt und damit übersehen werden. Es empfiehlt sich immer vor Beginn der OP, die gesunde Gegenseite klinisch zu untersuchen. So kann nach Platzierung der K-Drähte die Beweglichkeit sowie auch die Ellenbogenachse der betroffenen Seite (i.d.R. leichter Valgus) im Seitenvergleich beurteilt werden. Wenn eine Flexion von mindestens $120^{\circ}$ möglich ist, kann man davon ausgehen, dass keine wesentliche Rotationsfehlstellung vorliegt. Trotz aller dieser Möglichkeiten ist die intraoperative Überprüfung der regelrechten Stellung nicht immer einfach. Im seitlichen Strahlengang darf kein Rotationssporn (Kalibersprung, s. - Abb. 8) zu sehen sein. Die korrekte Stellung bez. der Kurvation wird mithilfe der Rogers-Hilfslinie (Verlängerung der ventralen Humeruskortikalis, die im Normalfall das Capitulum humeri am Übergang vom mittleren zum hinteren Drittel schneidet) bestimmt (s. - Abb. 9). Liegt der Schnittpunkt weiter ventral, besteht eine Antekurvationsfehlstellung, liegt der Schnittpunkt weiter dorsal, dann liegt eine Rekurvationsfehlstellung vor. Nach der K-Draht-Spickung schließt sich eine Ruhigstellung für 4 Wochen an. Nach radiologisch gesicherter Konsolidierung der Fraktur kann die ambulante Implantatentfernung im Rahmen einer Kurznarkose (bei perkutan eingebrachten K-Drähten in Analgosedierung) nach 5-6 Wochen durchgeführt werden. Als alternative Osteosynthesen stehen die mechanisch weniger stabile 2-fache radiale K-Draht-Spickung, die technisch anspruchsvolle, aber übungsstabile antegrade (absteigende) ESI-Nagelung (ESIN: elastisch stabile intramedul- 


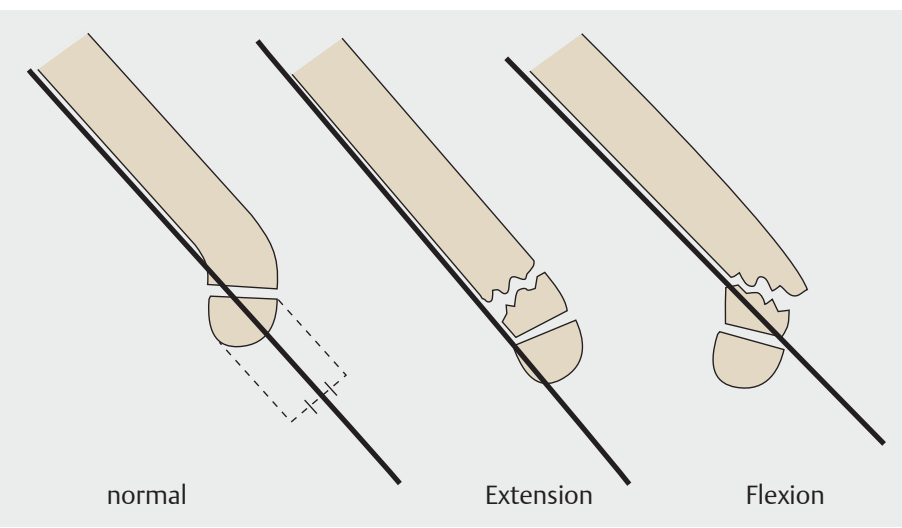

- Abb. 9 Rogers-Hilfslinie als Hinweis für die korrekte Stellung im seitlichen Strahlengang: a Bei korrekter Stellung, b bei Hyperextensionsstellung, c bei Hyperflexionsstellung. Quelle: von Laer L, Kraus R, Linhart W. Diagnostische Hinweise. In: von Laer L, Kraus R, Linhart W, Hrsg. Frakturen und Luxationen im Wachstumsalter. 6. Aufl. Stuttgart: Thieme; 2012: 145-149.

\section{Epikondyläre Abrissfrakturen}

\section{Verletzungsmechanismus/ klinisches Bild/Diagnostik}

Die Epikondylen des distalen Humerus sind sog. Apophysen, die metaphysär und damit extraartikulär liegen. Sie sind nicht am Längenwachstum beteiligt und dienen als Bandansatz und Muskelursprung. Der Verletzungsmechanismus ist meist ein Sturz auf den ausgestreckten Arm. In ca. 75\% der Fälle liegt eine Kombinationsverletzung mit einer Ellenbogenluxation vor, wobei es bei einem Drittel dieser Fälle bei Diagnosestellung bereits zur spontanen Reposition des Gelenks gekommen ist $[7,10]$. Bis auf sehr wenige Ausnahmen ist meist der ulnare Epikondylus betroffen.

Das klinische Bild ist davon abhängig, ob ein Luxationsmechanismus stattgefunden hat. Neben einer ausgeprägten Schwellung können sämtliche Befunde einer Gelenkluxation (u.a. deutliche Fehlstellung) einschließlich möglicher traumatischer Gefäß- und Nervenläsion vorliegen. Im luxierten Zustand ist die klinische Untersuchungsfähigkeit in aller Regel stark eingeschränkt.

Fixateur externe (cave N. radialis) zur Verfügung [1].

\begin{abstract}
Merke
Gelingt bei geschlossener Reposition keine anatomische Reposition der kindlichen suprakondylären Humerusfraktur, muss eine offene Reposition durchgeführt werden! Die intraoperative Stellungskontrolle beinhaltet die Bildwandlerkontrolle in 2 Ebenen und die Überprüfung der Extension und Flexion sowie der Ellenbogenachse im Seitenvergleich!
\end{abstract}

Neben der bereits beschriebenen Gefahr der iatrogenen Nervenschädigung vor allem des N. ulnaris (K-Draht-Spickung) und des N. radialis (Fixateur externe) besteht vor allem bei den Typ-III- und Typ-IV-Frakturen die Gefahr einer traumatischen Nervenläsion (i.d. R. Traktionsschaden) und/oder einer Gefäßläsion in der Ellenbeuge (A. brachialis). Nach langwierigen Repositionsversuchen und der teilweise ausgeprägten Schwellung muss immer ein Kompartmentsyndrom ausgeschlossen werden. Nicht korrekt reponierte und retinierte Frakturen führen zu entsprechenden Achs- und Rotationsfehlstellungen mit Einschränkungen der Ellenbogenbeweglichkeit und schicksalhaften u.a. auch kosmetisch sehr unangenehmen Wachstumsstörungen (Cubitus varus und valgus).

Nach Freigabe des Ellenbogengelenks bedarf es nahezu nie einer Physiotherapie. Die volle Beweglichkeit stellt sich quasi durch Eigenbeübung im Rahmen des kindlichen Spieltriebs individuell nach ca. 3-6 Wochen selbstständig ein. Die Sportfähigkeit besteht nach Erreichen der seitengleichen Ellenbogenbeweglichkeit.
Grundlage der Diagnosestellung ist die konventionelle Röntgenuntersuchung in 2 Ebenen. Schwierigkeiten bei der Interpretation der Röntgenbilder, die aufgrund der akuten Verletzung nahezu nie in dem korrekten a.-p. und seitlichen Strahlengang möglich sind, bereitet das relativ variable Erscheinen der entsprechenden Knochenkerne. Der Epicondylus ulnaris ist ab etwa dem 5. Lebensjahr, der des Epicondylus radialis ab dem 8.-13. Lebensjahr sichtbar. Da diese Kerne nicht selbst frakturieren, ist auf eine Erweiterung der knorpeligen Wachstumszone zwischen Apophysenkern und dem distalen Humerus zu achten. Vor allem ein fehlender Epicondylus ulnaris muss aufhorchen lassen. Hier liegt dann häufig eine Gelenkinterposition des Abrissfragmentes nach erfolgter Luxation vor (s. Abb. 10a-b). In einigen Fällen kann eine MRT (Magnetresonanztomografie) zur Sicherung der Diagnose und zur Beurteilung des übrigen Kapsel-Band-Apparates sowie zum Ausmessen der Dislokation sinnvoll sein.

\section{Merke \\ Bei Kindern ab dem 5. Lebensjahr muss bei fehlendem Epicondylus-ulnaris-Kern nach Ellenbogentrauma immer an das Vorliegen einer Luxationsfraktur ge- dacht werden!}

\section{Therapie/Komplikationen/Nachbehandlung}

Komplette Abrissfrakturen sind prinzipiell als instabile Verletzungen einzustufen und bedürfen bis zur Heilung einer entsprechend adäquaten Ruhigstellung. Eine Dehiszenz von bis zu $5 \mathrm{~mm}$, bei jungen Kindern sogar bis zu $10 \mathrm{~mm}$, kann belassen und der konservativen Therapie mit Oberarmcast zugeführt werden. Dabei sollte nach 

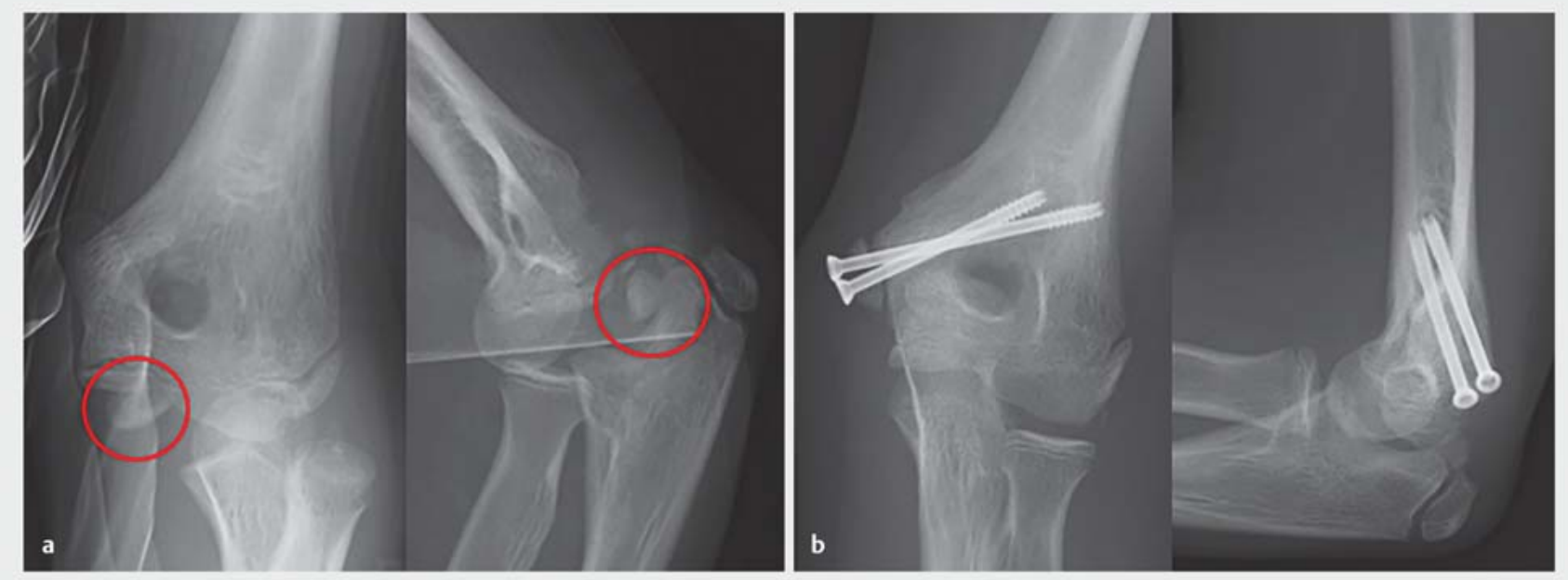

- Abb. 10 Ellenbogenluxation mit abgerissenem und interponiertem Epicondylus ulnaris (roter Kreis): a Präoperative Röntgenbilder in 2 Ebenen.

b Nach knöcherner Ausheilung nach offener Reposition und kanülierter Zugschraubenosteosynthese.

ca. 7 Tagen eine Röntgenverlaufskontrolle zum Ausschluss einer Sekundärdislokation erfolgen. Besteht eine weite Dislokation oder Rotation des Abrissfragments, wird die offene anatomische Reposition und wenn möglich übungsstabile Zugschraubenostesynthese (z. B. kanülierte Schrauben mit kurzem Gewinde) durchgeführt [4]. Bei sehr kleinen Kindern bzw. Epikondylenfragmenten kann es sein, dass nur eine K-Draht-Osteosynthese oder ggf. nur eine transossäre Naht möglich ist. In solchen Fällen bedarf es anschließend einer Schienenruhigstellung für 2-4 Wochen. Intraoperativ empfiehlt sich bei den Epicondylus-ulnaris-Frakturen zur sicheren Schonung die Darstellung des N. ulnaris. Eine vollständige Neurolyse ist nicht erforderlich. Nach Osteosynthese des Epikondylus muss intraoperativ die Stabilität des Ellenbogengelenks überprüft und bei Vorliegen z. B. einer zusätzlichen radialen Instabilität, vor allem bei Adoleszenten, eine zusätzliche Revision und entsprechende Versorgung des radialen Kapsel-Band-Apparates erfolgen.

\section{Merke}

Die operative Therapie der Wahl bei dislozierten epikondylären Frakturen am kindlichen distalen Humerus ist die offene anatomische Reposition und bewegungsstabile Zugschraubenosteosynthese.

Die häufigsten Komplikationen sind, bei unzureichender Ruhigstellung oder Retention, die Entwicklung einer klinisch nicht immer symptomatischen Pseudarthrose oder die Irritation des N. ulnaris [5].

Nach funktioneller Nachbehandlung (bei Zugschraubenosteosynthese) bzw. Ruhigstellung (bei konservativer Behandlung oder K-Draht-Osteosynthese) erfolgt jeweils nach ca. 4 Wochen eine Röntgenverlaufskontrolle und bei entsprechender Konsolidierung die Freigabe des Gelenks. Schrauben werden bei uns nach 12 und K-Drähte nach 6 Wochen im Rahmen eines ambulanten Eingriffs entfernt. Bei jüngeren Kindern ist in aller Regel keine Physiotherapie notwendig, bei Adoleszenten empfiehlt sich bei inadäquater Verbesserung der Beweglichkeit nach 34 Wochen die Durchführung von Krankengymnastik. Die Sportfähigkeit besteht ab dem Zeitpunkt der seitengleichen schmerzfreien Ellenbogenbeweglichkeit.

\section{Transkondyläre Frakturen}

\section{Verletzungsmechanismus/ klinisches Bild/Diagnostik}

Ursache sind meist Stürze auf den gestreckten Arm oder direkt auf den Ellenbogen. Diese Frakturen gehen durch die Meta- und Epiphyse und liegen somit intraartikulär. Überwiegend betroffen ist der Condylus radialis. Wesentlich seltener sind die Condylus-ulnaris- und Y-Frakturen, die meist Adoleszente betreffen, aber durch neuere Spielund Sportarten (z.B. Trampolinspringen, Bouldern) vermehrt auch jüngere Kinder betreffen.

Das klinische Bild ist wiederum abhängig vom Dislokationsgrad und kann daher sehr verhalten oder aber auch eindrücklich sein. Das diagnostische Standardverfahren ist die konventionelle Röntgenuntersuchung des Ellenbogengelenks in 2 Ebenen. Vollständig dislozierte Frakturen sind meist problemlos zu erkennen, bei jungen Kindern kann die Diagnose wegen des großen knorpeligen Anteils des Kondylus schwierig sein. Therapieentscheidend ist die sichere Unterscheidung zwischen der sog. hängenden (unvollständige Fraktur) und damit stabilen Fraktur von der vollständigen, noch undislozierten Fraktur, die 

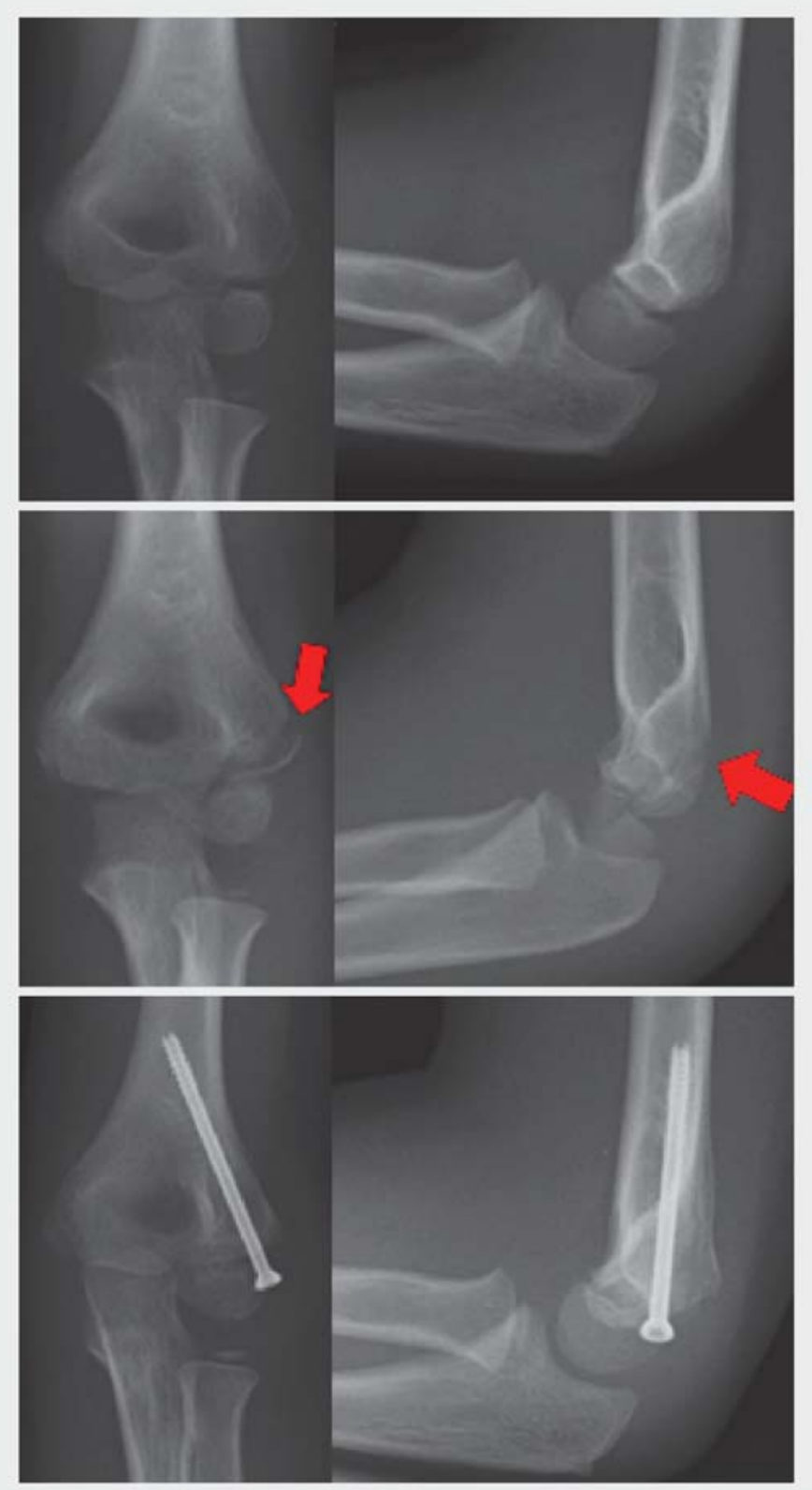

- Abb. 11 Röntgenbilder in 2 Ebenen von einer primär undislozierten augenscheinlich „stabilen“ Condylus-radialis-Fraktur bei einem 5-jährigen Kind; Nachweis einer Dislokation (rote Pfeile) bei der obligatorischen Kontrolle nach 5 Tagen sowie Röntgenbefund bei regelrechter Ausheilung nach offener Reposition und Zugschraubenosteosynthese.

als potenziell instabil interpretiert werden muss. Bei nicht dislozierten Frakturen ist deshalb nach primärer Ruhigstellung in der Oberarmschiene eine Röntgenverlaufskontrolle ohne Schiene nach 4-6 Tagen zwingend erforderlich, um eine sekundäre Dislokation auszuschließen. Zeigt sich dann auch nur eine minimale Dislokation, muss von einer Instabilität ausgegangen und damit eine OPIndikation gestellt werden.
Merke

Beim Vorliegen einer undislozierten Condylus-radialis-Fraktur ist die Röntgenverlaufskontrolle ohne Schiene nach 4-6 Tagen zum frühzeitigen Erkennen einer möglichen Dislokation zwingend erforderlich!

\section{Therapie/Komplikationen/Nachbehandlung}

Undislozierte stabile Frakturen werden in einem Oberarmdynacast für 4 Wochen ruhiggestellt. Dabei sollte, wie oben beschrieben, unbedingt nach 4-6 Tagen eine gipsfreie Röntgenkontrolle erfolgen, um eine Instabilität und Sekundärdislokation auszuschließen. Vollständige Frakturen des Condylus radialis neigen trotz adäquater Immobilisierung zur Sekundärdislokation und werden deshalb nicht umsonst als „Kadiläsion“ bezeichnet [7]. Instabile Frakturen, dislozierte Frakturen mit einer Dehiszenz von 2 mm oder mehr sowie Frakturen mit Gelenkstufe werden offen anatomisch reponiert und wenn möglich mittels übungsstabiler Zugschraubenosteosynthese retiniert (s. - Abb. 11). Bei sehr jungen Kindern oder sehr kleinen Fragmenten ist häufig nur eine K-Draht-Osteosynthese möglich, die anschließend einer additiven Ruhigstellung in einer Oberarmschiene für 2-4 Wochen bedarf. Die sehr seltenen Y-Frakturen oder transkondylären Frakturen treten meist bei älteren Kindern auf. Hier ist in aller Regel eine Plattenosteosynthese im Kombination mit Zugschrauben (oder K-Drähten) entsprechend der Erwachsenentraumatologie notwendig (s. A Abb. 12a-c) [2].

\section{Merke \\ Die operative Therapie der Wahl bei instabilen bzw. dislozierten transkondylären distalen Humerusfrak- turen beim Kind ist die offene anatomische Reposi- tion und bewegungsstabile Osteosynthese.}

Die häufigsten Komplikationen sind übersehene, meist sekundäre Fehlstellungen, die zu Pseudarthrosenbildung neigen. Da es sich um Gelenkfrakturen handelt, sind keine Korrekturen durch das Wachstum zu erwarten. Es kann typischerweise durch eine passagere Stimulation der Fuge bei ulnaren Frakturen zur Valgisierung, nach radialen und Y-Frakturen zur Varisierung der Ellenbogenachse kommen. Das Ausmaß dieser Varisierung ist abhängig von der Konsolidierungsdauer und somit von der Stabilität der Fraktur und dem angewandten Osteosyntheseverfahren [9].

Kompressionsschraubenosteosynthesen sind übungsstabil und können frühfunktionell nachbehandelt werden. Konservativ mittels Ruhigstellung behandelte Frakturen können nach radiologisch gesicherter Durchbauung nach ca. 4 Wochen freigegeben werden. In aller Regel bedarf es keiner Krankengymnastik. Bei anhaltenden Bewegungseinschränkungen nach knöcherner Konsolidierung oder bei älteren Kindern kann die Physiotherapie indiziert sein. Die Sportfähigkeit besteht nach Erreichen der freien 
Ellenbogenbeweglichkeit. Die elektiv ambulante Implantatentfernung erfolgt bei reiner K-Draht-Osteosynthese nach 5-6 und nach Schraubenosteosynthesen nach ca. 12 Wochen.

\section{Zusammenfassung/Prognose}

Frakturen am distalen Humerus sind die häufigsten Verletzungen des kindlichen Oberarms, wobei die typischen extraartikulären, metaphysären Frakturen von den intraartikulären, epiphysären Formen unterschieden werden müssen. Aufgrund der geringen Wachstumspotenz des distalen Humerus besteht selbst bei jungen Kindern ein sehr beschränktes Korrekturpotenzial für Fehlstellungen. Dies muss bei der Entscheidungsfindung zur Therapie dieser Frakturen bedacht werden. Instabile oder dislozierte Frakturen des distalen Humerus müssen beim Kind anatomisch reponiert und wenn möglich übungsstabil fixiert werden. Bei der am häufigsten vorkommenden suprakondylären Fraktur meist angewandten gekreuzten K-Draht-Spickung bedarf es, wie bei allen nur mit K-Draht-Spickung stabilisierten Frakturen am distalen Humerus, anschließend einer additiven Ruhigstellung. Die konsequente und regelrechte Therapie führt nachweislich zu exzellenten funktionellen Ergebnissen. Bei Frakturen am kindlichen distalen Humerus besteht immer die Gefahr für primär auftretende oder iatrogene Gefäßund/oder Nervenläsionen, die nicht übersehen werden dürfen und adäquat behandelt werden müssen. Primär nicht gesehene oder sekundär entstandene Dislokationen und Pseudarthrosenbildungen können das Outcome durch eine kosmetisch störende Fehlstellung, bleibende Bewegungseinschränkung, Schmerzen und Nervenirritation deutlich verschlechtern.

\section{Interessenkonflikt}

Die Autoren geben an, dass kein Interessenkonflikt besteht.

\section{Autorinnen/Autoren}

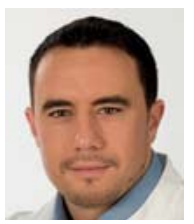

\section{Stefan Nuber}

Dr. med., Facharzt für Chirurgie, Orthopädie und Unfallchirurgie, spezielle Unfallchirurgie, Notfallmedizin; Oberarzt, Bereichsleiter Kindertraumatologie, Klinik für Unfallchirurgie, Orthopädie, Plastische und Handchirurgie, Klinikum Augsburg

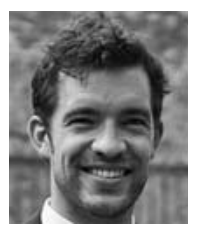

\section{Johannes Plath}

Dr. med.; Oberarzt, Klinik für Unfallchirurgie, Orthopädie, Plastische und Handchirurgie, Klinikum Augsburg

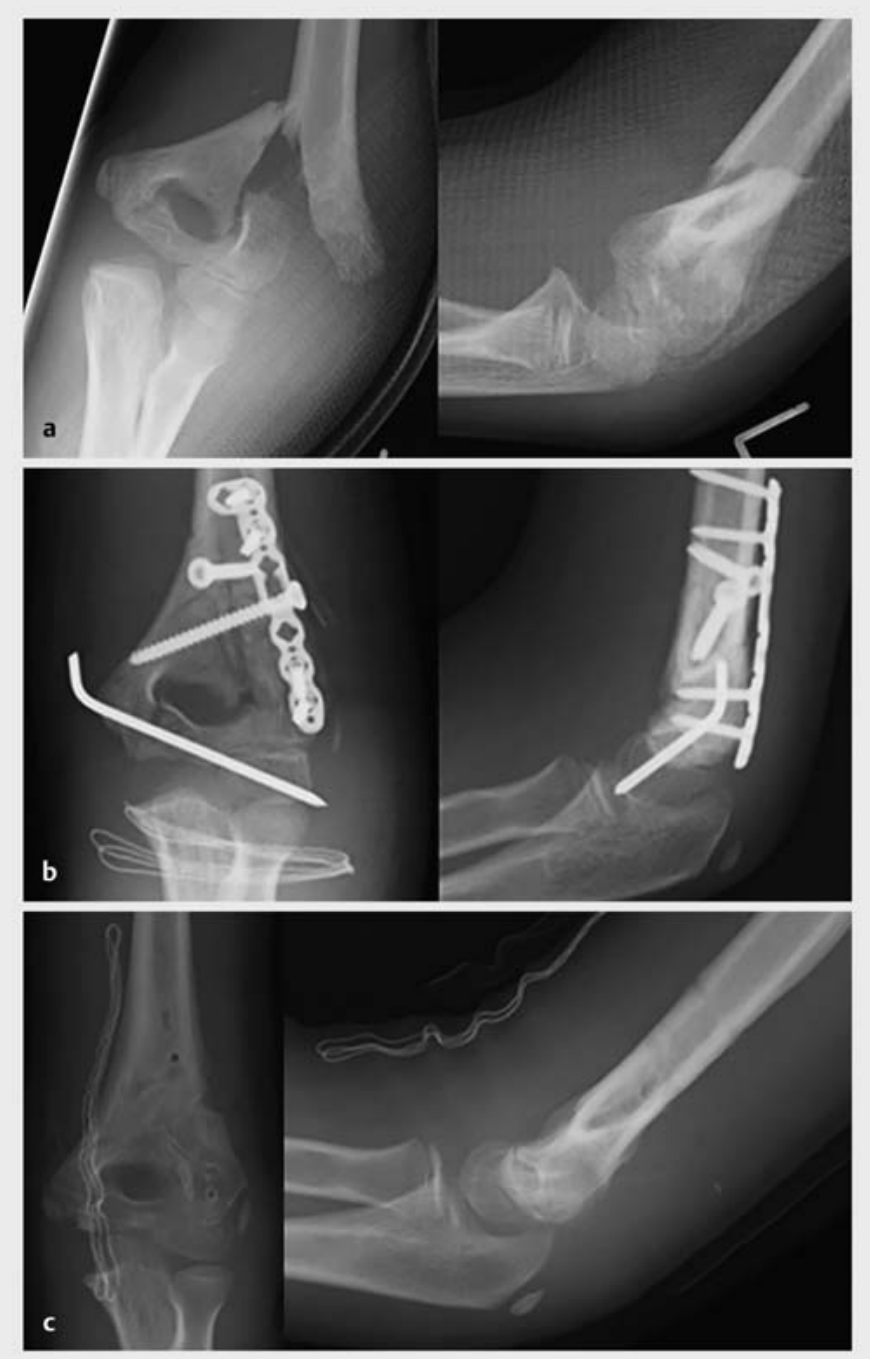

- Abb. 12 Transkondyläre Y-Fraktur bei einem 9-jährigen Mädchen, Röntgenbilder in 2 Ebenen vor (a) und nach (b) operativer Versorgung sowie nach Implantatentfernung nach regelrechter Ausheilung der Frak$\operatorname{tur}(\mathbf{c})$.

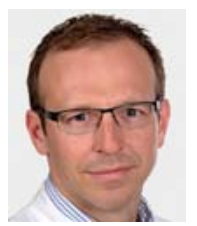

\section{Stefan Förch}

Dr. med.; Oberarzt, Klinik für Unfallchirurgie, Orthopädie, Plastische und Handchirurgie, Klinikum Augsburg

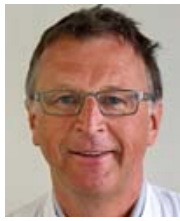

\section{Edgar Mayr}

Prof. Dr. med., Facharzt für Chirurgie, Orthopädie und Unfallchirurgie, spezielle Unfallchirurgie; Chefarzt, Klinik für Unfallchirurgie, Orthopädie, Plastische und Handchirurgie, Klinikum Augsburg 


\section{Korrespondenzadresse}

Dr. med. Stefan Nuber

Klinik für Unfallchirurgie, Orthopädie,

Plastische und Handchirurgie

Klinikum Augsburg

Stenglinstraße 2

86156 Augsburg

Tel.: 0821/400-2902

Fax: 0821/400-3313

stefan.nuber@klinikum-augsburg.de

Literatur

[1] AWMF online. S1-Leitlinie 012/2014: Suprakondyläre Humerusfraktur beim Kind. Im Internet: https://www.awmf.org/ leitlinien/detail/II/012-014.html; Stand: 03.12.2014, gültig bis 02.12 .2019

[2] AWMF online. S2 k-Leitlinie 006/126: Intraartikuläre Frakturen des distalen Humerus im Kindesalter. Im Internet: https://www.awmf.org/leitlinien/detail/ll/006-126.html; Stand: 31.03.2015, gültig bis 30.03.2020

[3] Babal JC, Mehlman CT, Klein G. Nerve injuries associated with pediatric supracondylar humeral fractures: a meta-analysis. J Pediatr Orthop 2010; 30: 253-263

[4] Case SL, Hennrikus WL. Surgical treatment of displaced medial epicondyle fractures in adolescent athletes. Am J Sports Med 1997; 25: 682-686
[5] Josefsson PO, Danielsson LG. Epicondylar elbow fractures in children. Acta Orthop Scand 1986; 57: 313-315

[6] Krauss R, Schneidmüller D, Röder C. Häufigkeit von Frakturen der langen Röhrenknochen im Wachstumsalter. Dtsch Ärztebl 2005; 102: A-838/B-708/C-661

[7] von Laer L, Kraus R, Linhart WE. Frakturen und Luxationen im Wachstumsalter. 6. Aufl. Stuttgart, New York: Thieme; 2012

[8] von Laer L, Günter SM, Knopf S et al. Die suprakondyläre Oberarmfraktur im Kindesalter - eine Effizienzstudie. Ergebnisse der multizentrischen Studie der Sektion Kindertraumatologie der Deutschen Gesellschaft für Unfallchirurgie. Teil II: Aufwand und Nutzen der Behandlung. Unfallchirurg 2002; 105: 217-223

[9] Marzi I, Hrsg. Kindertraumatologie. 2. Aufl. Berlin, Heidelberg, New York: Springer

[10] Weinberg AM, Marzi I, Günter SM et al. Die suprakondyläre Oberarmfraktur im Kindesalter - eine Effizienzstudie. Ergebnisse der multizentrischen Studie der Sektion Kindertraumatologie der Deutschen Gesellschaft für Unfallchirurgie. Teil I: Epidemiologie, Effektivitätsprüfung und Klassifikation. Unfallchirurg 2002; 105: 208-216

Bibliografie

DOI https://doi.org/10.1055/a-0594-5068

OP-JOURNAL 2018; 34: 279-288 @ Georg Thieme Verlag KC Stuttgart · New York ISSN 0178-1715 